\title{
Insuficiente adherencia al ejercicio físico de universitarios. Una revisión sistemática
}

\section{Insufficient adherence to the physical exercise of}

\author{
university students. A systematic review
}

\author{
Rubén Moreno Arrebola ${ }^{1 *}$, Pilar Puertas-Molero ${ }^{1}$, Carolina Castañeda-Vázquez ${ }^{2}$ y Manuel Castro-Sánchez ${ }^{1}$ \\ 1 Universidad de Granada (España). \\ 2 Universidad de Sevilla (España).
}

Resumen: El sedentarismo se constituye como uno de los principales problemas para la salud de la población mundial del siglo XXI. Debido a las características socioculturales que presentan los estudiantes universitarios, resulta una población idónea para el estudio y fomento de actividad física (AF). El objetivo principal de la presente revisión sistemática es dar una visión general sobre la adherencia a la práctica de AF entre la población universitaria, así como de los programas de intervención dirigidos a fomentar dicha práctica, desde el año 2007 hasta nuestros días. La metodología utilizada ha estado dirigida por la declaración PRISMA. Como principales conclusiones, el $100 \%$ de los estudios de la primera selección sostienen la urgente necesidad de la creación e implementación de programas de intervención dirigidos a fomentar la práctica de $\mathrm{AF}$ entre estudiantes universitarios. Finalmente, la mayoría de los programas de intervención estudiados consiguen incrementar la adherencia a la práctica de AF.

Palabras clave: Hábitos, actividad física, universidad, estudiantes, sedentarismo.
Abstract: A sedentary lifestyle is one of the main problems for the health of the world population of the 21st century. Due to the sociocultural characteristics presented by university students, it is a suitable population for the study and promotion of physical activity (PA). The main aim of the present systematic review is to give a general view on the adherence to the practice of PA among the university population, as well as intervention programs aimed at promoting this practice, from 2007 to the present day. The methodology used has been guided by the PRISMA statement. As main conclusion, $100 \%$ of the studies of the first selection support the urgent need for the creation and implementation of intervention programs aimed at promoting the practice of PA among university students. Finally, the intervention programs studied obtained good results, achieving in most of them an increase in the adherence to the practice of PA of the population studied.

Key words: Habits, physical activity, university, student, sedentarism.

\section{Introducción}

La Organización Mundial de la Salud (OMS, 2014) argumentan consistentemente que el sedentarismo es una de las principales lacras para la salud de la población mundial del siglo XXI, llegando a considerar este hábito como epidemia global. Según dicha organización, el sedentarismo se sitúa como cuarto factor de riesgo de mortalidad más importante a escala mundial, superado tan solo por la hipertensión, el consumo de tabaco y el exceso de glucosa en sangre. Según la OMS (2010) en el mundo mueren 1.9 millones de personas al año a causa de la inactividad física. El sedentarismo se asocia concretamente con muertes por cáncer (pulmonar, colon, esófago, mama, estómago y endometiro), enfermedades cerebrovasculares, hipertensión, enfermedades específicas de pulmón, enfermedades isquémicas del corazón, diabetes tipo 2, entre otras enfermedades cardiovasculares (ACSM, 2011). Para paliar esta situación, Zurita-Ortega et al. (2009) argumentan que existe un consenso generalizado entre profesionales de la salud que afirman que la práctica

Dirección para correspondencia [Correspondence address]:

Rubén Moreno Arrebola. Universidad de Granada (Espańa).

E-mail: rubenmorenoarrebola@gmail.com de AF regular es un factor decisivo para alcanzar un óptimo estado de salud.

De manera específica, el sedentarismo se define como "un modo de vida de poca agitación o movimiento” (RAE, 2015). Por otro lado, y de manera más concreta, la OMS (2010) define sedentarismo como el nivel de AF insuficiente, que se caracteriza por realizar menos de 150 minutos de AF a la semana. Siguiendo estas definiciones, el apego a la inactividad física se incrementa día a día. Las causas de este desapego son diversas. El avance tecnológico es uno de los principales causantes, aunque más que el avance tecnológico, es el mal uso de dicho avance. Cada día más, aparecen aparatos y maquinas que nos facilitan la vida, pero a su vez, nos restan práctica de AF y por ende salud. Prácticas diarias que hace unos años eran totalmente físicas, ahora con tan solo pulsar un botón están hechas. A priori, esto no debería ser un problema, si el tiempo que anteriormente se dedicaba a dichas tareas ahora se dedicara por ejemplo a practicar AF recreativa. Desgraciadamente, esto en la mayoría de los casos no ocurre, más bien sucede lo contrario, provoca apatía hacia la práctica de AF.

La calidad de vida relacionada con la salud se define como: "la percepción de un individuo de su posición en la vida, en el 
contexto cultural y el sistema de valores en que vive, en relación con sus metas, objetivos, expectativas, y preocupaciones" (Whoqol Group, 1995). Un gran número de estudios relacionan los hábitos saludables con una mejora calidad de vida. Concretamente una AF regular conlleva numerosos beneficios físicos, psicológicos y socio-afectivos (Zurita-Ortega et al, 2009). De esta forma, se sustenta que la práctica de AF, no solo ayuda a paliar determinadas enfermedades específicas, sino que ayuda a tener niveles de estrés más bajos y un nivel de vida saludable y un sentimiento de bienestar en general.

Las consecuencias negativas que conlleva el sedentarismo descritas anteriormente despiertan una especial necesidad de desarrollar diversos modelos de fomento de la práctica de AF, enfocados a poblaciones donde se tenga un impacto mayor. Según Blair y Hardman (1995), los grupos que se encuentran reunidos en ambientes institucionales son de especial interés. Si a esto se le ańade que la etapa universitaria está considerada como "frágil" por ser propensa a perder hábitos previos de AF (Corella, Rodríguez-Muñoz, Abarca-Sos y Zaragoza, 2017; García, 2001,), resulta una población idónea para la investigación y acción sobre la misma. El tránsito a la vida universitaria, conlleva consigo cambios muy importantes, llegando a ser un periodo de adaptación estresante debido a que se producen fuertes cambios en la vida de dichos sujetos. Esto implica, afrontar problemas con los que anteriormente nunca se habían topado. En este momento, existe un abandono parcial de los hábitos familiares, debido a cambios en la organización de tareas y de vida, gran oferta de comidas rápidas, falta de tiempo y estrés, inexperiencia al planificar una vida independiente, entre otros factores, (Papadaki, Hondros, Scott, \& Kapsokefalou, 2007). La etapa universitaria, por tanto, se sitúa como momento especialmente crítico para las personas, debido a que van a adquirir hábitos nuevos y reforzar otros (positivos o negativos) previos, que van a repercutir de manera significativa en su vida futura. Viendo el lado positivo de este cambio, y siguiendo a Wang et al. (2009) y a Chacón, Zurita, Ubago, González y Sánchez (2018), debemos aprovechar esta etapa, pues nos brinda unas condiciones idóneas para la adquisición de estilos de vida saludables. Por otro lado, no debemos olvidar que resulta de vital importancia que, desde etapas previas, (educación primaria y secundaria) traigan consigo hábitos de AF bien afianzados (González et al. 2017).

\section{Objetivos}

Aportar una visión general del estado de la cuestión actual sobre hábitos de AF de estudiantes universitarios y programas de intervención que fomentan dichos hábitos, impacto de las revistas donde se han publicado los trabajos y progresión de publicación a lo largo de la última década. Reunir conclusiones de los trabajos que miden el nivel de adherencia a la práctica de AF de la población universitaria que componen la primera muestra e instrumentos utilizados para medirla. Recabar información sobre los programas de intervención que se han llevado a cabo para mejorar la adherencia a los hábitos de $\mathrm{AF}$ en universitarios que han sido publicados en las dos bases de datos que consideramos WOS y SCOPUS.

\section{Método}

Enfocados a aportar la mayor rigurosidad y consistencia posible al presente trabajo, seguimos las directrices que marca la declaración PRISMA (Preferred Reporting Items for Systematic reviews and Meta Analyses) (Liberati et al. 2009). En este sentido, el diagrama de flujo del estudio es el siguiente:

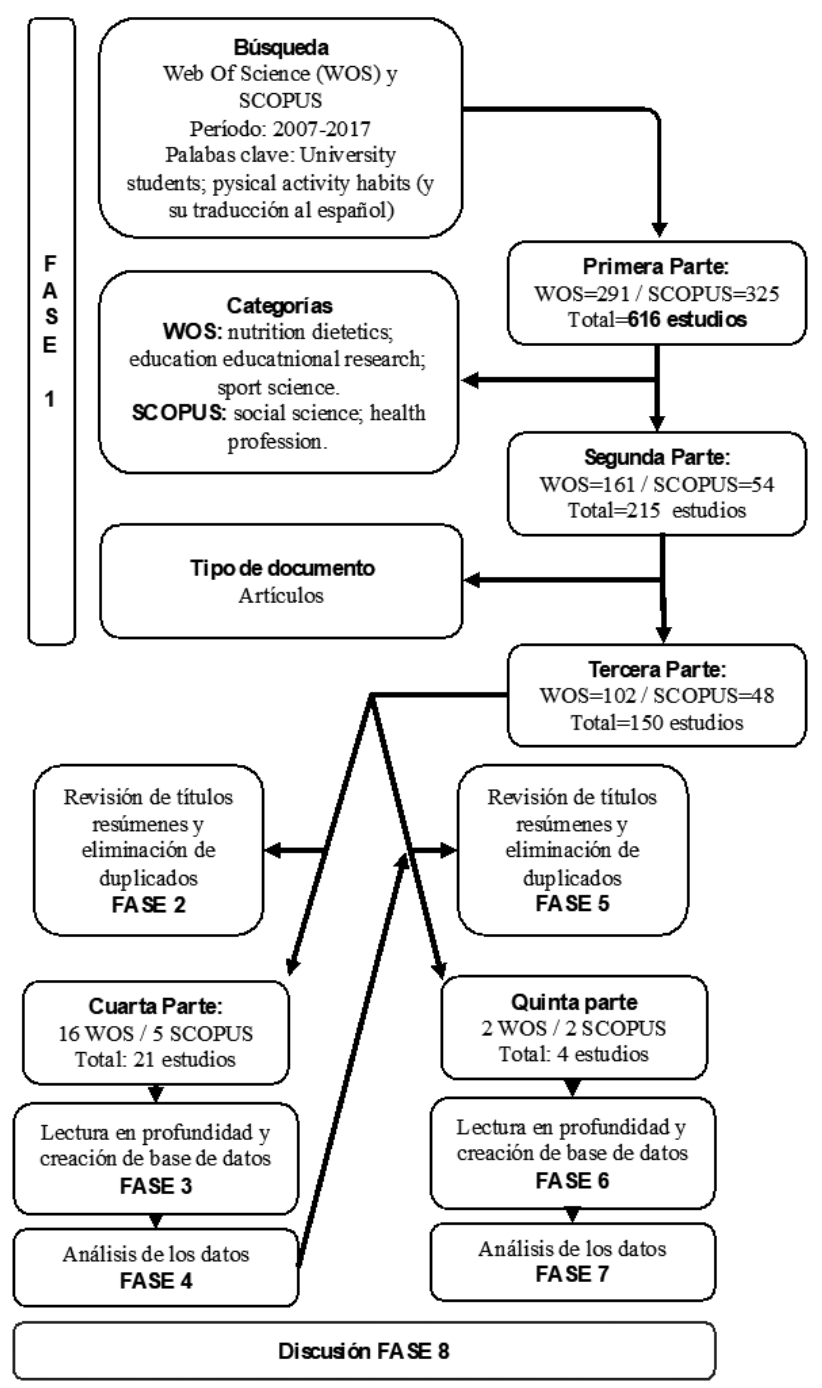

Figura 1. Diagrama de flujo del estudio. 


\section{Fases del estudio}

El estudio consta de dos procesos de revisión, con sus correspondientes fases. Del análisis de la primera revisión surge la segunda. La primera fase, la "búsqueda" es una fase común para ambos procesos de búsqueda. En ésta, se realiza una preselección de los trabajos científicos, utilizando únicamente los filtros presentes en las bases de datos utilizadas (WOS y SCOPUS). La segunda y quinta fase son las fases de selección “primera selección” y "segunda selección”. En ambas establecemos unos criterios de inclusión concretos. Para las mismas, se precisa la lectura de al menos los títulos y resúmenes de los trabajos preseleccionados. Estas fases se representan en la cuarta y quinta parte de la figura 1. La tercera y sexta fase son las fases de extracción de los datos, "primera extracción de datos" y "segunda extracción de datos". Para estas fases se hace necesaria una lectura pausada y exhaustiva de los artículos que han llegado hasta estas fases para crear una base de datos necesaria para las fases de análisis de los datos cuarta y séptima “primer análisis” y "segundo análisis". En éstas, analizamos la matriz de datos y presentamos los resultados. Por último, en la octava fase, discutimos los resultados obtenidos en ambos procesos de búsqueda, recogemos las conclusiones y limitaciones del presente estudio.

Búsqueda (fase 1)

Para este trabajo utilizamos como bases de datos Web of Science (WOS) y SCOPUS, pues se trata de las dos principales bases de datos para la temática que abordamos (Fernández-Revelles, 2014). Para esta primera fase, utilizaremos únicamente los filtros que nos aportan ambas bases de datos. Como palabras clave utilizamos "physical activity habits" y "university students" y como operadores booleanos "and". Se estableció un tramo temporal de una década, desde 2007 a 2017. Seguidamente, restringimos la búsqueda por área de conocimiento. En WOS se utilizaron nutrition dietetics, sport science y education educational research. En el caso de SCOPUS, utilizamos social science y health profession. Por último, acotamos la búsqueda por tipo de documento, incluyendo únicamente los artículos.

Selección (fase 2 y 5)

El presente trabajo consta de dos fases de selección, como ya hemos detallado anteriormente. Para el cribado de trabajos científicos en estas fases, se revisaron cuidadosamente los títulos y resúmenes, utilizando la información que nos reportan ambas bases de datos. Una vez seleccionados los artículos, se procedió a contrastar los trabajos duplicados en ambas bases de datos, y finalmente descargamos los textos completos de dichos trabajos.
La diferencia entre la fase 2 y la 5 son los criterios de inclusión. Para la primera selección (fase 2), se establecieron como criterios de inclusión los siguientes: trabajos cuantitativos transversales, que midieran el nivel de AF practicado en universitarios sanos, sujetos de edades iguales o superiores a 18 ańos, estudios que aportaran conclusiones sobre nivel de AF. Para la segunda selección (fase 5), nos centramos en trabajos de corte longitudinal (experimentales y cuasi-experimentales) que implementaran programas de intervención de fomento de la práctica de AF entre la población universitaria, implementados en una población de universitarios sanos, no incluimos programas específicos dirigidos a poblaciones muy reducidas de estudiantes con características muy concretas.

Extracción de los datos (fase 3 y 6 )

En estas fases, se requirió la lectura en profundidad de los artículos seleccionados, y se creó una base de datos para cada fase. (tabla 1).

Análisis de los datos (fase 4 y 7 )

Analizamos detalladamente los datos de las matrices de datos. Del análisis de la fase 4 surge todo el proceso siguiente, es decir, las fases desde la 5 a la 7.

Discusión (fase 8)

Discutimos los resultados, exponemos las principales conclusiones y recogemos las limitaciones del estudio.

Tras aplicar los primeros filtros (palabras clave y tramo temporal) obtenemos un total de 616 trabajos científicos referentes a la temática. Acotándola búsqueda por categorías, la muestra se redujo en 595 documentos, y en 65 fueron excluidos por no ser artículos. Al final de la fase 1 (búsqueda) obtuvimos una población total de 150 artículos para revisar (figura 1). Para la primera selección (fase 2), procedimos a revisar los títulos y resúmenes seleccionando únicamente aquellos documentos que cumplían con los criterios de inclusión descritos anteriormente. Tras esta selección, eliminamos los duplicados y descargamos el texto completo. De los 150 artículos revisados, 21 componen la muestra para esta primera selección. Para la segunda selección (fase 5), se lleva a cabo el mismo proceso que en la fase 2, pero utilizando criterios de inclusión diferentes. Al final de esta segunda selección, de los 150 artículos revisados, tan solo 4 cumplieron con los criterios de inclusión preestablecidos. Ambos procesos de selección se exponen detalladamente en la figura 1.

Insuficiente adherencia al ejercicio físico de universitarios. Una revisión sistemática SPORT TK, 8(1), 39-50 


\section{Resultados}

\section{Progresión e impacto de publicación en la última década}

En primer lugar, contemplamos como población del estudio, el total de documentos resultantes de la fase 1 (búsqueda) 150 artículos. Como se puede apreciar en la figura 2, la tendencia de publicación, tanto total como de ambas bases de datos, es ascendente, desde el año 2007 hasta nuestros días. Existiendo tan solo tres artículos de la temática en 2007 y llegando a su pico más alto en 2016 con un total de 26 estudios sobre el tema. Ambas bases de datos tienen la misma tendencia ascendente, aunque predominan las publicaciones en WOS.
Por un lado, la muestra de la primera selección, la componen el 14\% de los artículos de la población, el 10,6 \% pertenecen a WOS y el 3,33\% a SCOPUS. Se percibe una linealidad con la tendencia general, aunque existe un repunte de publicaciones seleccionadas del año 2010, siendo estos artículos los más antiguos para nuestra muestra 1. Para la misma muestra, el año con más publicaciones es 2016 con un total de cinco.

Por otro lado, la muestra de la segunda selección, está compuesta por el $2,66 \%$ de la población, perteneciendo el $1,33 \%$ a WOS e igualmente $1,33 \%$ a SCOPUS. La diferencia radica en que las publicaciones encontradas en WOS datan de 2017 y las recogidas de SCOPUS de 2012.

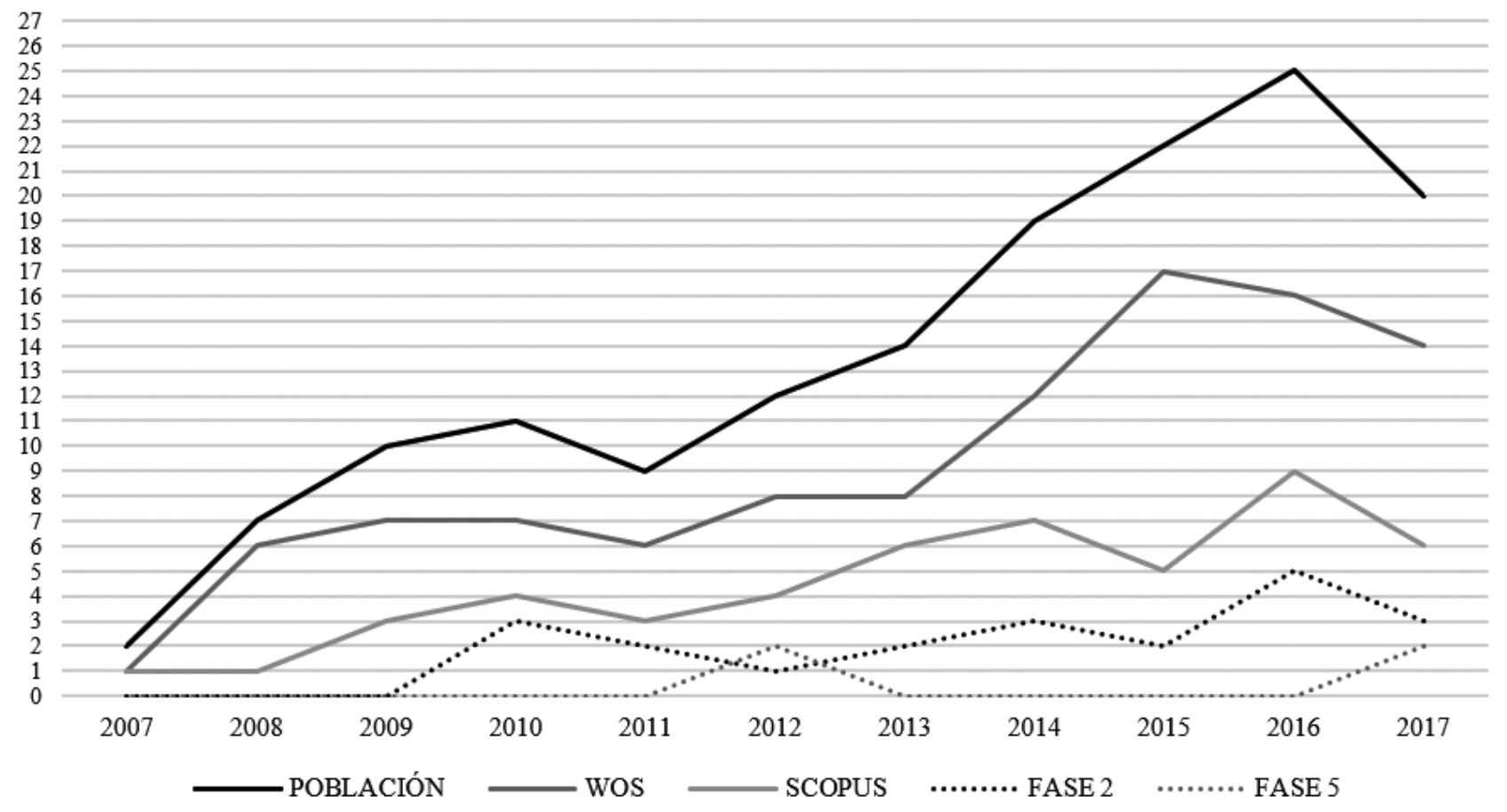

Figura 2. Progresión de publicación.

En cuanto al impacto, recogemos el h-index de las revistas donde han sido publicados los artículos seleccionados (tabla 1). Para la primera muestra, recogemos trabajos científicos de 14 revistas distintas. La revista de la que más trabajos hemos sustraído ha sido "Nutrición Hospitalaria" $(\mathrm{n}=5)$. Los índices de impacto de las mismas son muy heterogéneos, siete de las revistas tienen menos de 11 puntos, dos de ellas se encuentran entre 12 y 60 puntos y cuatro están por encima de 60 puntos. Para la segunda muestra, los trabajos provienen de tres revistas distintas. Los dos trabajos seleccionados en 2012, están publicados en "American Journal of Health Promotion" y los otros dos seleccionados en 2017 en revistas distintas. En cuanto al impacto, se encuentran entre un rango de 25 puntos y 78 .

En cuanto al idioma de publicación, para la primera muestra, predominan los artículos escritos en español ( $\mathrm{n}=14)$ frente a los escritos en inglés $(n=7)$. Los textos escritos en inglés gozan de los índices de impacto más altos, obteniendo una media de 69 puntos, frente a los 16 puntos de los artículos redactados en español. Para la segunda muestra, el 100\% de los artículos seleccionados están redactados en inglés, y obtienen una media de h-index de 60 puntos. 
Tabla 1. Datos de publicación e impacto.

\begin{tabular}{|c|c|c|c|c|}
\hline & Autores y año & Revista & Idioma & h-índex \\
\hline \multicolumn{5}{|c|}{ Impacto de las revistas de la primera selección (fase 2) } \\
\hline P1 & Da Cuña, Lantaron, González, y Gutiérrez (2017) & $\begin{array}{l}\text { Revista internacional de medicina y ciencias } \\
\text { de la actividad física y del deporte }\end{array}$ & Español & 10 \\
\hline $\mathrm{P} 2$ & $\begin{array}{l}\text { Castro-Sánchez, Linares-Manrique, San Román-Mata y Pérez } \\
\text { (2017) }\end{array}$ & $\begin{array}{l}\text { Sportis-scientific technical journal of school } \\
\text { sport physical education and psychomotricity }\end{array}$ & Español & - \\
\hline P3 & Cadarso, Dopico, Iglesias-Soler, Suárez, y Gude (2017) & Nutrición clínica y dietética hospitalaria & Español & 8 \\
\hline $\mathrm{P} 4$ & $\begin{array}{l}\text { Sevil, Práxedes, Abarca-Sos, Del Villar y García-González } \\
\text { (2017) }\end{array}$ & Nutrición clínica y dietética hospitalaria & Español & 8 \\
\hline $\mathrm{P} 5$ & Jiménez-Díaz, y Morera-Castro (2016) & $\begin{array}{l}\text { Mhsalud-revista en ciencias del movimiento } \\
\text { humano y la salud }\end{array}$ & Inglés & - \\
\hline P6 & Morgan y Elizondo (2016) & $\begin{array}{l}\text { Pensar en movimiento-revista de ciencias del } \\
\text { ejercicio y la salud }\end{array}$ & Español & - \\
\hline P7 & Alias, Aguilar y Hernández-Rodríguez (2016) & Psychology, Society and Education & Español & 4 \\
\hline P8 & Arboleda, Arango y Feito (2016) & RETOS & Español & 16 \\
\hline P9 & Wanden-Berghe, et al. (2015) & Nutrición hospitalaria & Español & 37 \\
\hline P10 & Gallardo-Escudero, Muñoz, Planells del Pozo, y López (2015) & Nutrición hospitalaria & Español & 37 \\
\hline P11 & Pérez, Lanío, Zelarayán, y Márquez (2015) & Nutrición hospitalaria & Español & 37 \\
\hline P12 & Pedišić, Rakovac, Bennie, Jurakić, y Bauman (2014) & Kinesiology & Español & 12 \\
\hline P13 & González, Díaz, Mendizábal-Ruiz, Medina, y Morales (2014) & Nutrición hospitalaria & Inglés & 37 \\
\hline P14 & Rodríguez et al. (2013) & Nutrición hospitalaria & Español & 37 \\
\hline P15 & $\begin{array}{l}\text { Dabrowska-Galas, Plinta, Dabrowska y Skrzypulec-Plinta } \\
\text { (2013) }\end{array}$ & Physical therapy & Inglés & 122 \\
\hline P16 & Moreno-Gómez, et al. (2012) & Public health nutrition & Inglés & 111 \\
\hline P17 & Castillo y Giménez (2011) & $\begin{array}{l}\text { Revista internacional de medicina y ciencias } \\
\text { de la actividad física y del deporte }\end{array}$ & Español & 10 \\
\hline P18 & Romaguera, et al. (2011) & Journal of sports sciences & Inglés & 102 \\
\hline P19 & Perusse-Lachance, Tremblay y Drapeau (2010) & $\begin{array}{l}\text { Applied physiology nutrition and metab- } \\
\text { olism-physiologie appliquee nutrition et } \\
\text { metabolisme }\end{array}$ & Inglés & 70 \\
\hline P20 & Gómez-López, Granero y Baena (2010) & Sports Science and Medicine & Inglés & 41 \\
\hline $\mathrm{P} 21$ & Vergaray, y Piérola (2010) & Apunts Medicina de l'Esport & Español & 7 \\
\hline \multicolumn{5}{|c|}{ Impacto de las revistas de la segunda selección (fase 5) } \\
\hline S1 & Cecchini-Estrada y Méndez-Giménez (2017) & European physical education review & Inglés & 25 \\
\hline S2 & Ashton, Morgan, Hutchesson, Rollo, y Collins (2017) & Nutrition journal & Inglés & 58 \\
\hline S3 & Greene, et al. (2012) & American Journal of Health Promotion & Inglés & 78 \\
\hline S4 & Hager, et al. (2012) & American Journal of Health Promotion & Inglés & 78 \\
\hline
\end{tabular}

Datos contextuales, poblacionales e instrumentos utilizados de las diferentes investigaciones

Con respecto a la localización de los estudios, se han encontrado trabajos que se han realizado en 10 países distintos. El país donde más se ha estudiado el tema es en España $(n=11)$, seguido de Costa Rica ( $\mathrm{n}=2)$. Es preciso resaltar, que existe una predominancia bastante acentuada, del 85,7\% de trabajos llevados a cabo en países de habla hispana.
En cuanto a la muestra participante en el total de trabajos seleccionados en la fase 2, suman un total de 16.908 sujetos. Con respecto al sexo, teniendo en cuenta a los trabajos donde se especifica este dato $(n=16)$, participan un total de 6012 hombres y 9188 mujeres. Las edades de los participantes oscilan entre medias de 19,78 y 24,15 años. Los instrumentos utilizados para medir el nivel de práctica de AF, predominan los cuestionarios Ad-hoc ( $\mathrm{n}=11)$. Por otro lado, el cuestionario más utilizado es el IPAQ (International Physical Activity 
Questionnaire), tres investigaciones utilizan su forma larga y uno su forma corta. Le sigue el GPAQ (Global Physical Activity Questionnaire) ( $\mathrm{n}=2$ ).

En la segunda muestra de estudios, los países donde se han implementado programas de intervención para fomentar la práctica de AF entre la población de estudiantes universitarios han sido 4, Estados Unidos ( $\mathrm{n}=2)$, Australia y España (tabla 2). Al contrario que en la muestra anterior, en este caso, predominan los países de habla anglosajona $(n=3)$ frente a los de habla hispana $(\mathrm{n}=1)$. La muestra de estudiantes total está compuesta por 5085 participantes, de los cuales 2942 son mujeres y 2143 son hombres. La edad de los participantes oscila entre medias de 19,1 y 22,1 años. Por último, en esta muestra los cuestionarios utilizados son tres. El IPAQ, predomina $(n=2)$ al igual que en la muestra anterior.

Tabla 2. Datos de la muestra e instrumentos de medida de la AF

\begin{tabular}{|c|c|c|c|c|c|c|}
\hline \multirow{2}{*}{ Código } & \multirow{2}{*}{ País } & \multicolumn{3}{|c|}{ Muestra } & \multirow{2}{*}{$\begin{array}{c}\text { Edad en años (media, } \\
\text { desviación típica o rango) }\end{array}$} & \multirow{2}{*}{ Instrumento de medida de la $\mathrm{AF}$} \\
\hline & & Mujeres & Hombres & Total & & \\
\hline \multicolumn{7}{|c|}{ Primera selección } \\
\hline $\mathrm{P} 1$ & España & - & - & 71 & - & Ad-hoc \\
\hline $\mathrm{P} 2$ & España & - & - & 61 & - & Ad-hoc \\
\hline P3 & España & 272 & 167 & 439 & $24,15 \pm 6$ & IPAQ \\
\hline $\mathrm{P} 4$ & España & 493 & 408 & 901 & $22.59 \pm 3.59$ & IPAQ-SF \\
\hline P5 & Costa Rica & 48 & 92 & 140 & $19,78 \pm 4.72$ & Ad-hoc \\
\hline P6 & Costa Rica & 109 & 97 & 206 & $21,99 \pm 1.55$ & PAQ \\
\hline P7 & España & 548 & 468 & 1011 & $21,98 \pm 4,68$ & Ad-hoc \\
\hline P8 & Colombia & - & - & 92 & - & GPAQ \\
\hline P9 & España & 1094 & 460 & 1753 & 21,35 & Ad-hoc \\
\hline $\mathrm{P} 10$ & España & 55 & $\ldots$ & 55 & $18-31$ & Ad-hoc \\
\hline P11 & Argentina & 273 & 281 & 554 & - & GPAQ \\
\hline $\mathrm{P} 12$ & Croacia & 783 & 471 & 1254 & $21,49 \pm 1.77$ & IPAQ \\
\hline $\mathrm{P} 13$ & México & 405 & 215 & 620 & 21 & NCHA-II \\
\hline P14 & Chile & 411 & 388 & 799 & $20,9 \pm 2.3$ & Ad-hoc \\
\hline P15 & Polonia & 236 & 64 & 300 & $20,94 \pm 1,03$ & IPAQ \\
\hline P16 & España & 538 & 449 & 987 & $21.5 \pm 3.3$ & Ad-hoc \\
\hline P17 & España & - & - & 975 & - & Ad-hoc \\
\hline $\mathrm{P} 18$ & España & 1188 & 863 & 2051 & $21,9 \pm 4,8$ & Encuesta nacional de salud de España \\
\hline P19 & Canadá & 1892 & 598 & 2490 & $24.1 \pm 5.4$ & Ad-hoc \\
\hline P20 & España & 843 & 991 & 1834 & - & $\begin{array}{l}\text { Questionnaire for the Analysis of Sports } \\
\text { Habits and Lifestyles }\end{array}$ \\
\hline $\mathrm{P} 21$ & Perú & - & - & 315 & 21 & Ad-hoc \\
\hline Total & & 6012 & 9188 & 16908 & & \\
\hline \multicolumn{7}{|c|}{ Segunda selección } \\
\hline S1 & España & 257 & 136 & 393 & $20,3 \pm 2,98(18-27)$ & IPAQ \\
\hline S2 & Australia & $\ldots$ & 50 & 50 & $22,1 \pm 2(18-25)$ & Godin Leisure-Time Exercise Questionnaire \\
\hline S3 & EEUU & 1047 & 624 & 1671 & $19.1 \pm 1.1(18-24)$ & IPAQ \\
\hline S4 & EEUU & 1638 & 1333 & 2971 & $19.9 \pm 2.6$ & PAR-Q (Physical activity readiness) \\
\hline Total & & 2942 & 2143 & 5085 & & \\
\hline
\end{tabular}


Programas de intervención implementados en la segunda muestra

En cada una de las investigaciones de esta muestra, se ha implementado un programa distinto. La duración de los programas oscila entre 10 y 15 semanas. Tan solo en una de ellas no se implementaron pautas de alimentación. Las intervenciones que han tenido lugar en 2017 llevan a cabo un tipo de formación mixto, compaginando teoría y práctica, frente a los programas implementados en 2012 que solo utilizaron formación teórica (tabla 3). Tres de los cuatro programas implementados obtienen una mayor adherencia a la práctica de $\mathrm{AF}$, mientras el otro, consigue mantener los niveles que ya tenían, evitando que estos bajen.

En el trabajo de Cecchini-Estrada y Méndez-Giménez (2017), se lleva a cabo un programa basado las estrategias TARGET que se centra en alentar a los estudiantes a desarrollar capacidades de autorregulación que les permitan adoptar hábitos de práctica de AF en su tiempo libre. Estas estrategias se basan directamente en el clima motivacional.

Ashton et al. (2017), en su trabajo llevaron a cabo el programa HEYMAN (Harnessing Ehealth to enhance Young men's Mental health, Activity and Nutrition). Este programa está enfocado expresamente para la población masculina de entre (18 y 25 años). El programa se sustenta en la teoría de la autodeterminación y en la teoría social cognitiva.

El programa "ProjectWebhelath", implementado en el trabajo de Greene et al. (2012), se centró únicamente en sesiones teóricas, impartidas a través de una web. Para el desarrollo curricular del programa se basaron en dos modelos educativos, el sistema de diseńo instructivo de y el modelo de motivación educativa.

Por último, Hager et al. (2012), llevaron a cabo el programa HERE (Health Education and Physical Education), basado en charlas presenciales u online, ofreciendo el mismo contenido en ambas.

Tabla 3. Programas implementados que fomentan la AF

\begin{tabular}{|c|c|c|c|c|c|c|}
\hline Código & Nombre & Diseño & Duración & Formación & $\begin{array}{c}\text { Pautas de } \\
\text { alimentación }\end{array}$ & $\begin{array}{c}\text { Resultados } \\
(p<0.05)\end{array}$ \\
\hline S1 & $\begin{array}{l}\text { Programa basado } \\
\text { en (TARGET) }\end{array}$ & Experimental & $\begin{array}{l}12 \text { semanas } \\
\text { (24sesiones) }\end{array}$ & $\begin{array}{l}\text { Teórica y } \\
\text { práctica }\end{array}$ & no & $\begin{array}{l}\text { Aumento de AF moderada y vigorosa } \\
\text { en el grupo experimental }\end{array}$ \\
\hline S2 & HEYMAN & Experimental & 12 semanas & $\begin{array}{l}\text { Teórica y } \\
\text { práctica }\end{array}$ & si & $\begin{array}{c}\text { Aumento de AF moderada y vigorosa } \\
\text { en el grupo experimental }\end{array}$ \\
\hline S3 & Project Webhealth & Experimental & 10 semanas & Teórica & si & $\begin{array}{l}\text { El grupo experimental, mantuvo los } \\
\text { niveles iniciales de actividad física. }\end{array}$ \\
\hline S4 & HEPE & Cuasi-experimental & 15 semanas & Teórica & si & $\begin{array}{l}\text { Aumento de AF moderada entre el pre } \\
\text { y el post }\end{array}$ \\
\hline
\end{tabular}

\section{Resultados y conclusiones de las investigaciones}

Resultados y conclusiones de las investigaciones de la primera búsqueda

En este sub-apartado, nos centramos exclusivamente en los resultados referentes al nivel de AF reportado por las investigaciones estudiadas y por otro lado en las conclusiones (tabla 4). En primer lugar, con respecto al nivel de AF reportado, tan solo el 28,5\% de los estudios exponen resultados donde el nivel de AF de los estudiantes encuestados es alto o moderado, frente al $71,5 \%$ de los trabajos, que reportan un nivel de AF que no llega a los mínimos recomendados. Dos de las cuatro investigaciones que reportan niveles altos de AF se llevaron a cabo a una población de estudiantes de carreras universitarias del campo de la salud como fisioterapia y medi- cina. De manera más específica, de los estudios que ofrecen el tanto por ciento de los estudiantes suficientemente activos e inactivos $(n=15)$, el 53,3\% de los trabajos recogen que más del $50 \%$ de su muestra de estudiantes universitarios, no cumplen con los mínimos recomendados. Las muestras de inactividad física expuesta por los trabajos objeto de estudio oscilan entre el $34 \%$ y el $81,2 \%$.

Para terminar este apartado, centrándonos en las conclusiones que ofrecen los artículos objeto de estudio, el 100\% de ellos (marcados con un si en la tabla 4), defienden y exigen una urgente necesidad de incrementar estudios sobre la temática y la creación e implementación de programas de intervención específicos que ayuden a fomentar la adherencia a la práctica de AF entre la población de estudiantes universitarios. Precisamente de este hallazgo, surge la segunda selección de estudios y el proceso que va desde la fase 5 a la fase 7. 
Tabla 4. Conclusiones sobre AF de la primera selección.

\begin{tabular}{|c|c|c|c|}
\hline Código & $\begin{array}{c}\text { Nivel de } \\
\text { AF }\end{array}$ & Resultados & $\begin{array}{l}\text { Necesidad de } \\
\text { más estudios } \\
\text { y programas }\end{array}$ \\
\hline $\mathrm{P} 1$ & alto & El índice de realización de AF en estudiantes de fisioterapia es elevado & $\mathrm{Si}$ \\
\hline P2 & insuficiente & Cinco de cada 10 estudiantes universitarios no realizan AF habitual & $\mathrm{Si}$ \\
\hline P3 & insuficiente & Cifras preocupantes en relación a las tasas de AF. & $\mathrm{Si}$ \\
\hline $\mathrm{P} 4$ & insuficiente & El 48,3\% cumplen con las recomendaciones de AF diaria, frente al 51,5\% que no las cumplen. & $\mathrm{Si}$ \\
\hline P5 & insuficiente & El $72 \%$ de los estudiantes presenta hábitos sedentarios. & $\mathrm{Si}$ \\
\hline P6 & insuficiente & El 81,2\% no cumplen con los mínimos recomendados. & $\mathrm{Si}$ \\
\hline P7 & insuficiente & El $62 \%$ de la muestra argumenta no practicar AF. & $\mathrm{Si}$ \\
\hline P8 & alto & El $51.1 \%$ de los estudiantes tienen un NAF alto, $26,1 \%$ moderado y un $22.8 \%$ bajo & $\mathrm{Si}$ \\
\hline P9 & insuficiente & Nivel de AF muy bajo & $\mathrm{Si}$ \\
\hline $\mathrm{P} 10$ & insuficiente & El 20\% son sedentarias y el 56,4\% una AF ligera (insuficiente) & $\mathrm{Si}$ \\
\hline P11 & alto & El 79.8\% de los estudiantes son físicamente activos & $\mathrm{Si}$ \\
\hline $\mathrm{P} 12$ & insuficiente & Bajos niveles de AF & $\mathrm{Si}$ \\
\hline P13 & insuficiente & El $37,2 \%$ son totalmente sedentarios & $\mathrm{Si}$ \\
\hline P14 & insuficiente & El nivel de inactividad física alto & $\mathrm{Si}$ \\
\hline $\mathrm{P} 15$ & alto & Los estudiantes de fisioterapia demostraron tener una adherencia del $100 \%$ a la práctica de AF. & $\mathrm{Si}$ \\
\hline P16 & insuficiente & El 46\% insuficientemente activos físicamente & $\mathrm{Si}$ \\
\hline $\mathrm{P} 17$ & insuficiente & EL 58,1\% de los estudiantes son insuficientemente activos físicamente & $\mathrm{Si}$ \\
\hline $\mathrm{P} 18$ & insuficiente & El 43\% de los estudiantes no practican AF, o la práctica de AF que realizan es insuficiente. & $\mathrm{Si}$ \\
\hline P19 & moderado & El 34\% de los estudiantes practican menos de 150 minutos semanales de AF. & $\mathrm{Si}$ \\
\hline P20 & moderado & El estudio se centra únicamente en los estudiantes que no realizan ningún tipo de AF (17,6\%) & $\mathrm{Si}$ \\
\hline $\mathrm{P} 21$ & insuficiente & El 42\% de los estudiantes no realizan AF moderada. & $\mathrm{Si}$ \\
\hline
\end{tabular}

Resultados y conclusiones de los programas de intervención

En los tres programas más recientes de 2017, obtienen como resultado una adherencia a la práctica de AF moderada y/o vigorosa en el grupo experimental con diferencias estadísticamente significativas $(p<0.05)$ con respecto al grupo control. $\mathrm{Y}$ uno de ellos obtiene que los niveles de AF se mantienen después de la implementación del programa.

Con respecto a las conclusiones, el estudio de CecchiniEstrada y Méndez-Giménez (2017), concluye que el desarrollo de capacidades de autorregulación de la práctica de AF de manera individual, fomenta la adherencia a la práctica de AF. Ashton et al. (2017), por su parte, cerciora que el programa HEYMAN demostró bastante potencial para impactar de manera positiva en una serie de resultados de salud, entre los que se encuentra el aumento de práctica de AF. Greene et al. (2012), concluyen que el programa ProjectWebhealth, tuvo un efecto positivo y perdurable en el tiempo, pues mantuvo los niveles iniciales de AF en una población, que si no hubiese participado habría experimentado una disminución significativa en este aspecto. Por último, Hager et al. (2012), argumentan que el programa HEPE influye positivamente en la adherencia a la práctica de AF de estudiantes universitarios y que el programa que se llevó a cabo de forma presencial, obtuvo mejores resultados que el impartido de forma virtual.

\section{Discusión}

Como objetivo principal de la investigación, nos marcamos dar una visión general del estado de la cuestión en la actualidad sobre la adherencia a la práctica de AF de estudiantes universitarios a escala mundial. Los resultados son preocupantes, y se evidencia que se trata de una población en riesgo importante de padecer problemas de salud (ACSM, 2011). Al igual que en las investigaciones de Cody et al. (2012), en nuestra investigación, se hace evidente que los estudiantes de carreras relacionadas con la salud, (medicina, fisioterapia, etc.) si cumplen con la AF mínima establecida por la OMS (2014).

En el presente estudio aparecen tasas preocupantes de inactividad física para la población de estudiantes universitarios, al igual que en el estudio de Irwin (2004), concluía 
que, a escala mundial, entre el 30 y $60 \%$ de los estudiantes en etapa universitaria no practican suficiente AF. MorenoArrebola, Fernández-Revelles, San Román, y Espejo (2018) en su revisión sobre el mismo tema, recogen que el 50\% de los trabajos estudiados reportan insuficiente AF por parte de los estudiantes universitarios. Además, existen investigaciones como la de Haase, Steptoe, Sallis, y Wardle (2004) que aportan datos incluso más preocupantes, pues argumentan, en su estudio en países europeos, que la inactividad física de los estudiantes universitarios oscila entre el 35 y 89\% de dicha población. Por el contrario, Corella et al. (2017) aportan resultados favorables al respecto en su estudio sobre el cumplimiento de las recomendaciones de AF en estudiantes universitarios españoles, concluyendo que un gran porcentaje de estos cumplió con las recomendaciones de AF moderada.

En el presente estudio se hace patente por diversos estudios (Gallardo-Escudero et al., 2015; Pérez et al., 2014; Rodríguez et al., 2013; Sevil, 2016) la necesidad de fomentar la creación e implementación de programas de intervención enfocados a mejorar la adherencia a la práctica de AF de estudiantes universitarios. Al respecto, hemos encontrado en las bases de datos de referencia, la implantación de tan solo cuatro programas. Sabemos que existen programas de nivel internacional, enfocados específicamente para dar recomendaciones de práctica de AF, como, por ejemplo, las "Recomendaciones mundiales sobre AF para la salud" (OMS, 2014) o el manual del Departamento de salud, AF, mejora y protección de la salud del Reino Unido (2011), titulado "Comenzar activo, mantenerse activo". La propuesta que hacemos desde esta investigación es la creación de programas específicos que alternen directrices teóricas y prácticas, basados en teorías que se han contrastado eficaces para fomentar la práctica de AF entre universitarios, por ejemplo, trabajar formas de motivación más autodeterminadas (Alias et al., 2016; Sevil et al., 2016), mejora de las capacidades física básicas (Gómez-López et al., 2010) disminuir la grasa corporal y conseguir un equilibrio energético (Gómez, Ceballos y Enríquez, 2018) o crear entornos propicios para el cambio de hábitos saludables en el contexto universitario (Romaguera et al. 2011) y en programas como los explicitados anteriormente, donde se establecen unas directrices claras a seguir. Es preciso que esto no quede solo en la teoría y vaya más allá llevándose a la práctica real, que se lleven a cabo proyectos de investigación de este tipo y que se le dé visibilidad para dar la máxima extensibilidad a los hallazgos, para poder ir mejorando y puliendo determinados aspectos.

\section{Conclusiones}

El incremento de práctica de AF en estudiantes universitarios se trata de un tema novedoso y con un creciente interés a nivel mundial en la última década, especialmente en países de habla hispana, (España y países de Centro América y Sudamérica). En cuanto a los programas de intervención para fomentar la práctica de AF entre la población estudiada, existe un creciente interés por el tema, aunque aún las investigaciones al respecto son escasas. El tema en general, aglutinando ambas muestras, goza de una gran extensibilidad e impacto.

Existe una fuerte tendencia a la creación de herramientas Ad-hoc, en su mayoría cuestionarios, para medir la práctica de AF en universitarios. El cuestionario más utilizado para medir dicha adherencia a la práctica de AF en ambas muestras es el IPAQ (International Physical Activity Questionnaire). El $100 \%$ de los trabajos revisados de la primera muestra argumentan la necesidad inminente de la creación e implementación de programas dirigidos a fomentar la práctica de AF entre estudiantes universitarios.

Con respecto a los programas investigados, la duración de los mismos es muy similar oscilando entorno a los 3 meses de implementación. El tipo de formación que predomina en los programas implementados más recientemente, combinan formación teórica y práctica y obteniendo los mejores resultados de adherencia a la práctica de AF. De manera general, en los programas investigados, se obtienen buenos resultados, consiguiendo en la mayoría de las ocasiones un incremento de la práctica de AF prolongada en el tiempo.

\section{Limitaciones del estudio}

Los resultados que aportamos no son generalizables.

Al centrarnos únicamente en dos bases de datos, probablemente estemos dejando fuera estudios relacionados con el tema objeto de estudio, que podrían aportar información relevante.

\section{Referencias bibliográficas}

1. Alias, A., Aguilar, J. M., \& Hernández-Rodríguez, A. I. (2016). Motivaciones de los estudiantes universitarios ante la práctica de actividad físico-deportiva de tiempo libre. Las actividades náuticas. Psychology, Society, \& Education, 8(3), 229-242.

2. American College of Sports Medicine [ACSM]. (2011). Quantity and Quality of Exercise for Developing and Maintaining Cardiorespiratory, Musculoskeletal, and Neuromotor Fitness in Apparently Healthy Adults: Guidance for Prescribing. Exercise Medicine \& Science in Sports \& Exercise, 43(7):1334-1359.
3. Arboleda, V. H., Arango E. F., \& Feito, Y. (2016). Actividad física y percepciones de beneficios y barreras en una universidad colombiana. $R E$ TOS. Nuevas Tendencias en Educación Física, Deporte y Recreación, (30), 15-19.

4. Ashton, L. M., Morgan, P. J., Hutchesson, M. J., Rollo, M. E., \& Collins, C. E. (2017). Feasibility and preliminary efficacy of the 'HEYMAN' healthy lifestyle program for young men: a pilot randomized controlled trial. Nutrition journal, 16(1), 1-17.

5. Blair, S. N., \& Hardman, A. (1995). Physical Activity, Health, and 
Well-Being-An International Scientific Consensus Conference-Quebec City, May 19-21, 1995-Introduction.

6. Cadarso, A., Dopico, X., Iglesias-Soler, E., Suárez, C., \& Gude, F. (2017). Calidad de vida relacionada con la salud y su relación con la adherencia a la dieta mediterránea y la actividad física en universitarios de Galicia. Nutrición Clínica y Dietética Hospitalaria, 37(2), 42-49.

7. Castillo, E., \& Giménez, F. J. (2011). Hábitos de práctica de actividad física del alumnado de la Universidad de Huelva. Revista Internacional de Medicina y Ciencias de la Actividad Física y del Deporte/International Journal of Medicine and Science of Physical Activity and Sport, 11(41), 127-144.

8. Castro-Sánchez, M., Linares-Manrique, M., Sanromán-Mata, S., \& Pérez, A. J. (2017). Análisis de los comportamientos sedentarios, práctica de actividad física y uso de videojuegos en adolescentes. Sportis. Scientific Journal of School Sport, Physical Education and Psychomotricity, 3(2), 241-255.

9. Cecchini-Estrada, J. A., \& Méndez-Giménez, A. (2017). Motivational climate, $2 \times 2$ achievement goal orientation and dominance, self-regulation, and physical activity in pre-service teacher education. European Physical Education Review, 23(4), 461-479.

10. Chacón, R., Zurita, F., Ubago, J.L., González, G., \& Sánchez, M. (2018). Condición física, dieta y ocio digital según práctica de actividad física en estudiantes universitarios de Granada. SPORT TK: revista euroamericana de ciencias del deporte, 7(2), 7-12.

11. Cody, F., Durkin, M. W., Blair, S. N., Keller C., Beth, M., \& Rast, J. (2012). Determining levels of physical activity in attending physicians, resident and fellow physicians and medical students in the USA. British Journal of Sports Medicine, 46(5), 360-364.

12. Corella, C., Rodríguez-Muńoz, S., Abarca-Sos, A., \& Zaragoza, J. (2017). Cumplimiento de las recomendaciones de práctica de actividad física en función de los cutoffs points y el género en estudiantes universitarios españoles. SPORT TK: Revista Euroamericana de Ciencias del Deporte, 7(1), 9-18.

13. Da Cuña, I., Lantarón, E. M., González, Y., \& Gutiérrez, M. (2017). Repercusión del sedentarismo en la respuesta cardiorrespiratoria en estudiantes universitarios. Revista Internacional de Medicina y Ciencias de la Actividad Física y del Deporte/International Journal of Medicine and Science of Physical Activity and Sport, 17(66), 367-378.

14. Dabrowska-Galas, M., Plinta, R., Dabrowska, J., \& Skrzypulec-Plinta, V. (2013). Physical activity in students of the Medical University of Silesia in Poland. Physical therapy, 93(3), 384-392.

15. Department of Health, Physical Activity, Health Improvement and Protection, United Kingdom (2011). Star Active, Stay Active: A report on physical activity from the four home countries' Chief Medical Officers. Extraído de: http://www.dh.gov.uk/en/Publicationsandstatistics/ Publications/PublicationsPolicyAn dGuidance/DH_128209

16. Gallardo-Escudero, A., Muñoz Alférez, M. J., Planells del Pozo, E. M., \& López Aliaga, I. (2015). La etapa universitaria no favorece el estilo de vida saludable en las estudiantes granadinas. Nutrición Hospitalaria, 31(2), 975-979.

17. García, M. (2001). Los españoles y el deporte: prácticas y comportamientos en la última década del siglo XX. Consejo Superior de Deportes. Ministerio de Educación, Cultura y Deportes. Madrid.

18. Gómez, E., Ceballos, O., \& Enríquez, M.C. (2018). Nivel de actividad física, equilibrio energético y exceso de peso en jóvenes universitarios. SPORT TK: Revista Euroamericana de Ciencias del Deporte, 7(2), 101-108.

19. Gómez-López, M., Granero, A., \& Baena, A. (2010). Perceived barriers by university students in the practice of physical activities. Journal of sports science \& medicine, 9(3), 374-381.

20. González, G., Zurita, F., Puertas, P., Espejo, T., Chacón, R., \& Castro, M. (2017). Influencia de los factores sedentarios (dieta y videojuegos) sobre la obesidad en escolares de Educación Primaria. Reidocrea, 6(11), 120-129.
21. González, C. E., Díaz, Y., Mendizábal-Ruiz, A. P., Medina, E., \& Morales, J. A. (2014). Prevalencia de obesidad y perfil lipídico alterado en jóvenes universitarios. Nutrición Hospitalaria, 29(2), 315-321.

22. Greene, G. W., White, A. A., Hoerr, S. L., Lohse, B., Schembre, S. M., Riebe, D., ... \& Blissmer, B. (2012). Impact of an online healthful eating and physical activity program for college students. American Journal of Health Promotion, 27(2), 47-58.

23. Haase, A., Steptoe, A., Sallis, J. F., \& Wardle, J. (2004). Leisure-time physical activity in university students from 23 countries: associations with health beliefs, risk awareness, and national economic development. Preventive medicine, 39(1), 182-190.

24. Hager, R., George, J. D., LeCheminant, J. D., Bailey, B. W., \& Vin cent, W. J. (2012). Evaluation of a university general education health and wellness course delivered by lecture or online. American Journal of Health Promotion, 26(5), 263-269.

25. Irwin, J. (2004). Prevalence of university students' sufficient physical activity: A systematic review. Perceptual and Motor Skills, 98(3), 927943.

26. Jiménez-Díaz, J., \& Morera-Castro, M. (2016). Desempeńo motor y hábitos de actividad física en estudiantes universitarios en Costa Rica. MHSalud, 12(2), 15-24

27. Liberati, A., Altman, D. G., Tetzlaff, J., Mulrow, C., Gøtzsche, P. C., Ioannidis, J. P., ... \& Moher, D. (2009). The PRISMA statement for reporting systematic reviews and meta-analyses of studies that evaluate health care interventions: explanation and elaboration. PLoS medici$n e, 6(7)$, e1000100.

28. Moreno-Arrebola, R., Fernández-Revelles, A.B., San Román, S., \& Espejo, T. (2018). Revisión sistemática sobre hábitos de actividad física en estudiantes universitarios. Sportis. Scientific Journal of School Sport, Physical Education and Psychomotricity, 4(1), 162-183.

29. Moreno-Gómez, C., Romaguera-Bosch, D., Tauler-Riera, P., BennasarVeny, M., Pericas-Beltran, J., Martínez-Andreu, S., \& Aguilo-Pons, A. (2012). Clustering of lifestyle factors in Spanish university students: the relationship between smoking, alcohol consumption, physical activity and diet quality. Public health nutrition, 15(11), 2131-2139.

30. Morgan, S. J., \& Elizondo, J. H. (2016). Actividad física y otros hábitos de vida saludables de estudiantes de medicina de la Universidad de Costa Rica. Pensar en Movimiento: Revista de Ciencias del Ejercicio y la Salud, 14(1), 1-14.

31. Organización Mundial de la Salud [OMS]. (2010). La Organización Mundial de la Salud y el Comité Olímpico Internacional firman un acuerdo para fomentar los modos de vida sanos. Recuperado de: http:// www.who.int/mediacentre/news/releases/2010/ioc_20100721/es/

32. Organización Mundial de la Salud. (2014). Recomendaciones sobre actividad física para la salud. Ginebra: Ediciones de la Organización Mundial de la Salud.

33. Papadaki, A., Hondros, G., Scott, J. A., \& Kapsokefalou, M. (2007). Eating habits of university students living at, or away from home in Greece. Appetite, 49(1), 169-176.

34. Pedišić, Ž., Rakovac, M., Bennie, J., Jurakić, D., \& Bauman, A. E. (2014). Levels and correlates of domain-specific physical activity in university students: cross-sectional findings from Croatia. Kinesiolo$g y, 46(1), 12-22$.

35. Pérez, G., Lanío, F. A., Zelarayán, J., \& Márquez, S. (2014). Actividad física y hábitos de salud en estudiantes universitarios argentinos. Nutrición Hospitalaria, 30(4), 896-904.

36. Perusse-Lachance, E., Tremblay, A., \& Drapeau, V. (2010). Lifestyle factors and other health measures in a Canadian university community. Applied Physiology, Nutrition, and Metabolism, 35(4), 498-506.

37. Real Academia de la Lengua Espańola de la Lengua (2015). Diccionario de la Lengua Española. Madrid: Espasa.

38. Rodríguez, R., Palma, L., Romo, B., Escobar, B., Aragú, G., Espinoza, O., ... \& Gálvez, C. (2013). Hábitos alimentarios, actividad física y 
nivel socioeconómico en estudiantes universitarios de Chile. Nutrición Hospitalaria, 28(2), 447-455.

39. Romaguera, D., Tauler, P., Bennasar, M., Pericas, J., Carlos Moreno, C., Martinez, S., \& Aguilo, A. (2011) Determinants and patterns of physical activity practice among Spanish university students. Journal of Sports Sciences, 29(9), 989-997.

40. Sevil, J., Práxedes, A., Abarca-Sos, A., Del Villar, F., \& García-González, L. (2016). Levels of physical activity, motivation and barriers to participation in university students. The Journal of sports medicine and physical fitness, 56(10), 1239-1248.

41. Vergaray, J. J. B., \& Piérola, J. W. Z. (2010). Los futuros médicos y la actividad física: una contradicción no saludable. Apunts. Medicina de l'Esport, 45(167), 151-159.

42. Wanden-Berghe, C., Martín-Rodero, H., Rodríguez-Martín, A., Novalbos-Ruiz, J. P., Martínez de Victoria, E., Sanz-Valero, J., ... \& Irles,
J.A. (2015). Calidad de vida y sus factores determinantes en universitarios españoles de Ciencias de la Salud. Nutrición Hospitalaria, 31(2), 952-958.

43. Wang, D., Ou C.Q., Chen M.Y., \& Duan N. (2009) Health-promoting lifestyles of university students in Mainland China. BMC Public Health 9, 379 .

44. Whoqol Group. (1995). The World Health Organization quality of life assessment (WHOQOL): position paper from the World Health Organization. Social science \& medicine, 41(10), 1403-1409.

45. Zurita-Ortega, F., Fernández-García, R., Cepero-González, M., Zagalaz-Sánchez, M., Valverde-Cepeda, M., \& Ramírez-Domínguez, P. (2009). The relationship between pain and physical activity in older adults that begin a program of physical activity. Journal of Human Sport and Exercise, 4(3), 248-297. 